\title{
Privilegierte Internationalisierung exklusiver Internatsschulen?
}

\author{
Ulrike Deppe
}

Online publiziert: 9. Juni 2020

(C) Der/die Autor(en) 2020

Zusammenfassung Während im gymnasialen Segment eine steigende Internationalisierung festgestellt und Schule insgesamt zunehmend unter transnationalen Bedingungen thematisiert wird, wurden Internatsschulen in Deutschland bislang in Bezug auf ihren Umgang mit globalisierten und transnationalen Bedingungen und mit Internationalisierungsprozessen nicht untersucht. Anhand der vergleichenden Analyse von Schulleiterinterviews exklusiver staatlicher und privater Internatsschulen sowie unter Rückgriff auf ethnografische Beobachtungen sowie Selbstdarstellungen der Schulen wird im Beitrag untersucht, wie die Schulleiter Internationalität und Internationalisierung verstehen und sich in Bezug auf Prozesse der Internationalisierung und Transnationalisierung entwerfen und diese bearbeiten.

Im Ergebnis lassen sich unterschiedliche Modi herausarbeiten, in denen Internationalität und Internationalisierung einen Teil der Legitimationspraxis und Superioritätsbehauptung der Schulen darstellen, als Schulentwicklungsaufgabe bearbeitet und als Rekrutierungsressource genutzt werden. Die Art der Trägerschaft ist jedoch eine zentrale Bedingung für die Ausgestaltung der Entwürfe und Praktiken der Internationalisierung und Transnationalisierung.

Schlüsselwörter Internatsschulen · Internationalisierung · Transnationalisierung · Wettbewerb · Privilegierung 


\section{Privileged internationalisation of German exclusive boarding schools?}

Abstract While the German secondary-level school segment is growingly "being international" and "globalised", and schools as a whole are increasingly being addressed under transnational conditions, boarding schools in Germany have so far not been no examined with regard to how they deal with globalised and transnational conditions and internationalisation processes. Based on the comparative analysis of interviews with school principals at exclusive state-run and private boarding schools, as well as drawing on ethnographic observations and the schools' self-representations, the article examines how the schools understand internationality and internationalisation, and how they draft and deal with processes of internationalisation and transnationalisation. As a result, different modes can be identified in which internationality and internationalisation are represented as a part of the legitimation practices and superiority claims of the schools, are processed as school development tasks, and are used as recruiting resources. The sponsorship, however, is a central condition for the design of the drafts and practices of internationalisation and transnationalisation.

Keywords Boarding schools · Competition · Internationalisation · Privilege · Transnationalisation

\section{Einführung}

Im gymnasialen Segment wird eine steigende Internationalisierung festgestellt (vgl. Helsper et al. 2016, S. 706; Zymek 2016) und Schule wird insgesamt zunehmend unter transnationalen Bedingungen thematisiert und untersucht (vgl. Adick 2018; Hornberg 2018; Hinrichsen und Paz 2018; Hummrich 2018; Pfaff 2018). So haben fast alle Gymnasien mindestens eine Partnerschule in einem anderen Land und führen Schüleraustausche durch. Zunehmend werden mehrsprachige Curricula und Abschlüsse an Gymnasien angeboten (vgl. Zymek 2016). Dabei bietet der Begriff „Internationalität“ eine Projektionsfläche, an die gleichzeitig eine Vielzahl von sehr unterschiedlichen und auch widersprüchlichen Argumenten und Agenden anknüpfen kann. Dadurch erhält der Begriff eine scheinbare Selbstverständlichkeit, die nicht mehr in jedem Einzelfall gerechtfertigt werden muss und dadurch als wirksame Quelle der Legitimierung und Distinktion erscheint (vgl. Waldow 2018). Internatsschulen wurden in Deutschland hingegen insgesamt wenig (vgl. Gibson 2017) und darüber hinaus gar nicht in Bezug auf Bedingungen, Anforderungen sowie ihren Umgang mit Prozessen der Internationalisierung, Transnationalisierung und Globalisierung untersucht. In dem Beitrag wird die Frage bearbeitet, wie sich exklusive Internatsschulen diesbezüglich positionieren und damit umgehen.

Im Folgenden werden der aktuelle Forschungsstand und die vorliegenden theoretischen Ansätze zur Globalisierung, Internationalisierung bzw. Transnationalisierung von Schule zusammengefasst, um anschließend die Ergebnisse der komparativen Analyse der Internatsschulen aus dem Sample der Studie „Biografische Verläufe und Berufskarrieren von Absolventinnen und Absolventen exklusiver Internatsgym- 
nasien in Deutschland“ vorzustellen. Diese werden schließlich in die bestehenden Erkenntnisse zu exklusiven Gymnasien, zu internationalen Schulen und Schulprogrammen sowie Studien zu Elite-Schulen in der internationalen Forschungsliteratur eingeordnet. Anschließend werden weiterführende Überlegungen und theoretische Anschlüsse skizziert. Dabei werden aus dem englischsprachigen Forschungsstand die Zuschreibungen und Bezeichnungen jener Schulen als „Elite“ übernommen, zugleich aber nicht auf den deutschen Forschungsstand bzw. die beforschten Einrichtungen übertragen.

Wenn im Weiteren von „exklusiven“ Bildungseinrichtungen die Rede ist, handelt es sich um Schulen mit einem gymnasialen Bildungsgang im deutschen Bildungssystem, die sich zwar in privater wie in staatlicher Trägerschaft befinden können, jedoch durch Eignungstests, verpflichtende Gespräche, Auswahlverfahren und/oder ökonomische Zugangshürden ihre Plätze einer ausgewählten Klientel vorbehalten und sich damit einer freien Anwahl oder wohnortbezogenen Kriterien verschließen bzw. diese ausschließen (vgl. ausführlich Deppe und Kastner 2014). Im deutschsprachigen Raum ist eine weiterführende Elitebildung solcher Einrichtungen im Sinne einer gewissen Langlebigkeit der Einrichtungen, stabilen und signifikanten Erfolgen in den Abschlussexamen, dem Eintritt in prestigeträchtige Universitäten und Fakultäten oder dem fortwährenden Einmünden der Absolvent*innen in leitende gesellschaftliche Positionen nicht nachgewiesen (vgl. Kenway et al. 2013, S. 18). Dieser Nachweis wäre aber zu führen, um die Einrichtungen wie im englischsprachigen Raum als „Elite“ klassifizieren zu können (vgl. Gaztambide-Fernández 2009). Dementsprechend sind bei den Bezugnahmen auf Eliteschulen ausschließlich Einrichtungen im anglophonen und französischen Sprachraum gemeint, bei denen diese o.g. Verbindungen bekannt sind.

\section{Forschungsstand zur Internationalisierung höherer Schulen}

Der Beitrag schließt an mehrere Forschungslinien an: Erstens weisen Studien zu exklusiven Gymnasien nach, dass diese Internationalisierung und Internationalität in ihre Semantiken einfließen lassen sowie internationale Curricula und Programme verstärkt anbieten (vgl. Helsper et al. 2016; Hornberg 2018; Kotzyba et al. 2018). Diese Forschungen stellen auch in nicht genuin internationalen Schulen Internationalisierungsprozesse fest. Gymnasien, die nun internationale, mehrsprachige Curricula und Abschlüsse anbieten, nehmen deutlich zu (vgl. Deppe et al. 2018a). Das International Baccalaureat (IB) nimmt durch seine Verbreitung insbesondere in der gymnasialen Oberstufe inzwischen eine besondere Stellung ein (s. Abb. 1; vgl. Deppe et al. 2018a; Hornberg 2018). Daneben gibt es das Cambridge International General Certificate of Education, das ebenfalls zunehmend an deutschen Schulen angeboten wird.

Die Zertifizierung der Schulen durch die Vergabeorganisationen, wie z. B. der International Baccalaureate Organization (IBO), ist kostenintensiv, dementsprechend gibt es immer noch mehr private als staatliche Schulen, die das IB oder das Cambridge Certificate anbieten. Aber auch letztere nehmen deutlich zu (vgl. Hornberg 2018). Die Besonderheit für staatliche Gymnasien liegt im Vergleich zu privaten 


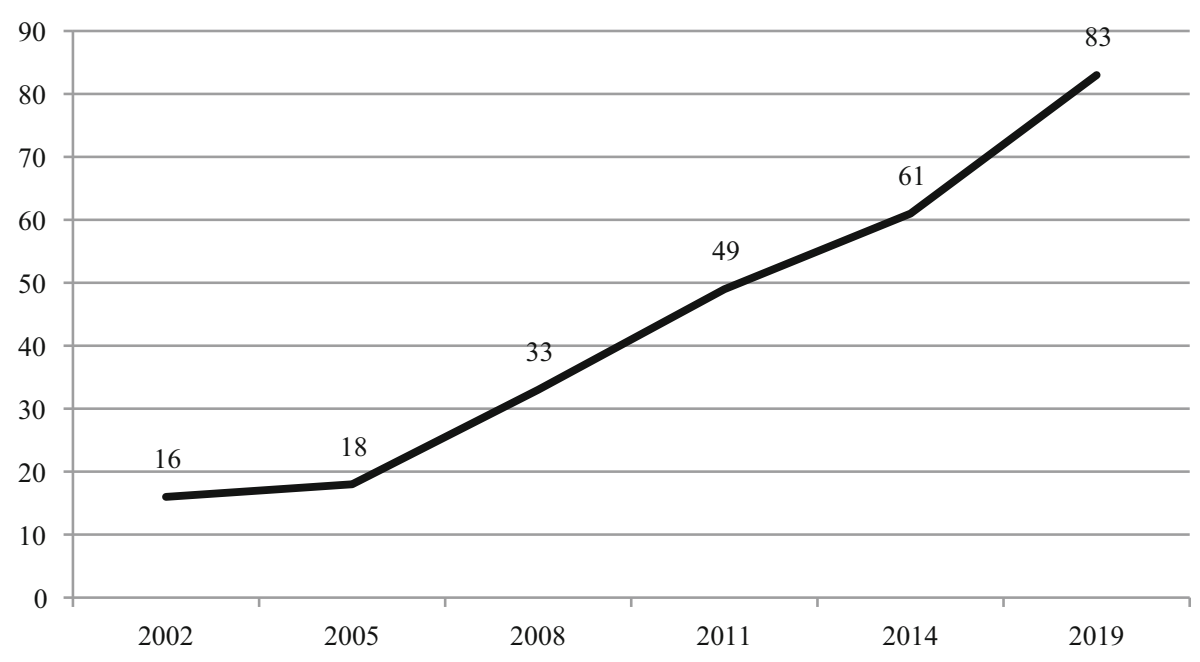

Abb. 1 Anzahl IB-Diploma-Programme an Schulen in Deutschland nach Jahren (Quelle: Eigene Berechnungen auf der Datengrundlage der International Baccalaureate Organization 2019)

Schulen darin, dass die Schüler*innen nicht zwischen Abitur und dem international anerkannten Abschluss wählen, sondern nur entscheiden können, ob sie zusätzlich den international anerkannten Abschluss ablegen möchten. Auch wenn von Überschneidungen zwischen den Fächern für die Schülerschaft ausgegangen wird, erhöht sich das Pensum an Arbeitszeit und die Prüfungen. Das gilt auch für das AbiBac, eine staatlich angebotene und damit wenig kostenintensive Möglichkeit, gleichzeitig das französische Baccalaureat und das deutsche Abitur abzulegen.

In der zweiten anzuknüpfenden Forschungslinie handelt es sich um Studien zur Expansion internationaler (und europäischer) Schulen. Diesen Forschungen zufolge wenden sich zudem in Deutschland dauerhaft sesshafte Familien verstärkt dem internationalen Schulsegment zu (vgl. Keßler und Krüger 2018; Hornberg 2018). Neben der Verteilung und Etablierung im deutschsprachigen Raum zeigen die Arbeiten die weltweite Verbreitung des IB sowie die zunehmende Ausdifferenzierung internationaler Schulen (vgl. Hayden und Thompson 2016; Helsper et al. 2016; Resnik 2016; Hornberg 2018; Keßler und Krüger 2018; Kotzyba et al. 2018; Krüger et al. 2019). Internationale Schulen weisen große Unterschiede in der Programmatik, den Zielgruppen, der geopolitischen Ausrichtung und ihrer Orientierung bzw. Gebundenheit an nationale Bildungssysteme, rechtlichen Bedingungen und den Organisationsformen auf (vgl. Hornberg 2010; Hayden und Thompson 2016). Hornberg (2018) charakterisiert internationale Schulen, aber auch Schulen mit internationalen Programmen wie dem IB in Anschluss an Adick (2008) als transnationale Bildungsräume, da sie durch das Operieren auf einer inter- und supranationalen Ebene jenseits staatlicher Curricula und Zertifikate liegen (vgl. Pfaff 2018).

Eine dritte Forschungslinie beschäftigt sich mit dem Zusammenhang der Globalisierung von Bildungsmärkten und Stratifizierungsprozessen innerhalb nationaler Bildungssysteme. Die Studien arbeiten heraus, dass Internationalisierungsprozesse im deutschen Bildungswesen nicht nur eine Reaktion auf die Globalisierung der 
(Bildungs-) Märkte darstellen, sondern ebenfalls als Ausdruck und Gegenstand von Stratifizierungsprozessen innerhalb des Bildungswesens zu betrachten sind (vgl. Zymek 2016; Deppe et al. 2018a; Kotzyba et al. 2018; Pfaff 2018). Den Ergebnissen zufolge führen diese zu einer Hierarchisierung von Transnationalität und Internationalität (vgl. Deppe et al. 2018a; Yildiz 2018; Keßler et al. 2018; Pfaff 2018). Im Segment exklusiver Schulen werden dann Internationalisierung und Transnationalisierung als „Motor sozialer und globaler Mobilität sowie als inhaltliche Innovation“ (Pfaff 2018, S. 162) gelesen, während Schulen mit hohem Migrationsanteil generell durch öffentliche, aber auch wissenschaftliche Diskurse als stigmatisiert und benachteiligt gekennzeichnet werden (vgl. Pfaff 2018; Deppe et al. 2018a).

Viertens kann an eine Forschungslinie aus dem anglophonen Raum zu sog. Elite-Internatsschulen und deren Globalisierungsstrategien angeschlossen werden (vgl. Brooks und Waters 2015; Courtois 2016; Howard 2018). Kenway et al. (2017) belegen, dass sich diese Internatsschulen in einem globalen Internatsschulmarkt verorten und konkurrieren, wohingegen die Lokalisierung in der örtlichen Gemeinde die Aktivitäten der Schüler*innen und das Alltagsgeschäft der Schule betreffen (vgl. auch Brooks und Waters 2015; Courtois 2016; Howard 2018; Maxwell 2018). Zugleich wird deutlich, dass das Vorbild der britischen Internatserziehung oder auch das IB koloniale Logiken in der Etablierung lokaler Eliten sowie der Aufrechterhaltung globaler Machtverhältnisse über transnationale Bildungsräume fortschreiben (vgl. Pfaff 2018; Prosser 2018; Angod und Gaztambide-Fernández 2019).

Wie exklusive Internatsschulen in Deutschland mit Internationalisierung umgehen bzw. wie diese von den Schulen entworfen und verstanden wird, wurde bisher nicht erforscht. Zwar liegen einzelne Studien zu Internatsgymnasien und ihren Schulkulturen vor (vgl. Kalthoff 1997; Böhme 2000; Helsper et al. 2001; Gibson 2017; zsmf. Deppe 2019), diese untersuchen die Schulen jedoch nicht in Hinblick auf die Fragestellung.

\section{Theoretische Ansätze und Begriffe}

Für die Beschreibung und Erklärung der Phänomene, in denen Schulen Beziehungen und Interaktionen in nationalstaatsübergreifenden Zusammenhängen intensivieren und pflegen und zugleich durch eine zunehmend multinationale Schülerschaft geprägt sind, existieren je nach Fokus unterschiedliche Begriffe wie Internationalisierung, Transnationalisierung und Globalisierung.

Globalisierung ist ein Begriff, der ursprünglich den Austausch von Ideen, Politiken und Gütern auf globaler Ebene beschreibt. Die Globalisierung der Bildung wurde daher als die Tendenz zum Isomorphismus verstanden, die in der weltweiten Schulversorgung und Bildungspolitik zu beobachten ist, sowie in einer zunehmenden Kommodifizierung der Bildung auf der ganzen Welt (vgl. Ball 2012; Spring 2015). Insbesondere Forschungen im Kontext des Neoinstitutionalismus belegen die Verbreitung eines bestimmten, modernen Bildungssystems mit Ähnlichkeiten auf formaler wie inhaltlicher Ebene, und vermuten die Existenz eines globalen Normenwerks, das nationale und lokale Politiken und Akteure beeinflusst (vgl. Meyer 2005). Kritiken am Neoinstitutionalismus weisen darauf hin, dass wichtige Unter- 
schiede auf der lokalen Ebene durch den Ansatz nicht erklärt werden können und nicht berücksichtigt wird, wie lokale Akteure globale Normen interpretieren und ihre eigenen, unterschiedlichen Praktiken entwickeln (vgl. Boli 2006; Deppe et al. 2018b, S. 4f.).

Kritiken an der Weltgesellschaftstheorie betonen auch, dass nicht nur Ideen, Güter und Politiken weltweit zirkulieren, sondern dass auch die Arbeitskräfte zunehmend global mobil sind (vgl. Bauman 1998b). Obwohl sich diese Gruppen weniger mit einer Nation identifizieren, bleiben sie dennoch oft mit der Familie und ihren lokalen Gemeinschaften verbunden. Aus diesem Grund wurde der Begriff „Transnationalisierung“ (vgl. Ong 1999; Pries 2010) eingeführt, um zu erfassen, wie Gruppen von Menschen und Organisationen nicht nur in Bezug auf Mobilität und Reichweite transnational werden, aber auch um zu erforschen, welche Formen sozialer Ungleichheit sich auf transnationaler Ebene herausbilden (vgl. Weiss 2005; Deppe et al. 2018b). Dementsprechend betrachtet der Transnationalisierungs-Ansatz gewissermaßen die Globalisierung ,von unten“ (vgl. Guarnizo und Smith 1998), indem er die nicht-staatlichen Akteure, die nicht mehr nur einem nationalstaatlichen, sondern durch die Wanderungsbewegungen mehreren Kontexten verbunden sind, in den Blick nimmt.

Die Forschung zur Globalisierung von Bildung hat sich auf die Vermarktung, Korporatisierung und Neoliberalisierung von Politiken und Praktiken konzentriert (Bellmann 2008; Ball 2012). Solche Analysen erfordern einen Fokus auf die Vernetzung zwischen den globalen, internationalen, nationalen und lokalen Märkten, politischen Kontexten und Akteuren (Bauman 1998a; Stronach 2010). Dementsprechend fokussieren sich Konzepte von Internationalisierung auf zwischenstaatliche Strukturen und Programme, die auf Kooperationen, Vereinbarungen und Transaktionen basieren, wobei nationalstaatliche Akteure miteinander in Austausch treten (vgl. Pfaff 2018). Knight (2004), deren Arbeit sowohl in der Hochschulforschung als auch in anderen Bildungsstufen weitreichend rezipiert wurde, beschreibt Internationalisierung als den Prozess der ,Integration einer internationalen, interkulturellen oder globalen Dimension in den Zweck, die Funktionen oder die Durchführung der nachschulischen Bildung“ (Knight 2004, S. 9). Diese Definition ist zwar neutral, wodurch sich einerseits ihre Anwendbarkeit in verschiedenen Kontexten erweitert, weshalb andererseits aber auch die Gründe für Internationalisierung, der Wert solcher Prozesse und die spezifischen Ergebnisse aus dem Blick geraten (vgl. Yemini 2015, S. 20). Die Definition ist überdies unspezifisch, da sie die Beziehung zwischen den drei genannten Dimensionen der Internationalisierung nicht berücksichtigt (vgl. Yemini 2015). Parallel findet sich im Feld der Schule die dominante Begriffsverwendung der Internationalität (vgl. Hinrichsen und Paz 2018). Als Grund dafür wird vorrangig die Vagheit gesehen, mit der sich an unterschiedliche Bildungsprinzipien und -versprechen anknüpfen lässt, nämlich einerseits den Erfordernissen eines globalen Wissensmarktes im Sinne von Humankapital und andererseits dem humanistisch konnotierten Ideal eines ,,world citizen“ zu entsprechen (vgl. Yemini 2015; Keßler et al. 2018; Waldow 2018).

An dieser Stelle wird auf die Begriffsentwicklungen von Adick (2008) und deren Weiterentwicklungen rekurriert, indem zwischen internationalen und transnationalen Bedingungen und Prozessen differenziert wird (vgl. Hummrich 2018). Damit 
wird die Frage fokussiert, wie sich die Einzelschulen in der Auseinandersetzung mit transnationalen Prozessen und Bedingungen positionieren (vgl. Hummrich 2018; auch Ullrich 2014). Denn während Internationalisierung und Transnationalisierung von Bildung bislang vorwiegend vorangetrieben durch transnationale Bildungsanbieter, staatliche und supranationale Organisationen thematisiert wurden, kann dies jedoch nicht die unterschiedlichen Formen der Internationalisierung und Transnationalisierung an Einzelschulen bzw. deren Umgang erklären, noch welche Funktion Internationalisierung und Transnationalisierung in einem Schulsystem einnimmt, in welchem sich immer stärker lokale und regionale Disparitäten in der sozialen Zusammensetzung bei gleichzeitiger Konkurrenz um die passenden Schüler*innen abzeichnen (vgl. Helsper et al. 2015; Deppe et al. 2018a, 2018b).

Deshalb wird das Verständnis von Internationalisierung und Transnationalisierung erweitert, indem diese als stratifizierender Prozesse und international gerahmte Bildung als stratifikatorische Ressource in Anschluss an Weber (2002) verstanden werden (Kotzyba et al. 2018). Dabei wird zwischen positiv und negativ privilegierendem Status unterschieden und von einer Interdependenz zwischen diesen Positionen ausgegangen. Wenn die Internationalisierung und Transnationalisierung der Bildung und ihre Wirkung auf die soziale Ordnung in ein Verhältnis gesetzt werden, können besondere Formen formaler Bildung wie international gerahmte Bildung als distinktive Markierer des Konsums, eines besonderen Lebensstils und zugleich als „legitimatorische Ressource“ (Waldow 2018, S. 248) fungieren. Damit sind die Überlegungen anschlussfähig an ein Verständnis einer Kommodifizierung von Bildung im Kontext globalisierungstheoretischer Ansätze. Die anbietenden Organisationen, Angebote und Schulen bedienen eine ,global middle class“ (vgl. Ball und Nikita 2014; Kenway et al. 2017; Kotzyba et al. 2018), aber eben auch eine lokale Mittelschicht, die auf Statusgewinne und den Zugang zu einem globalen Arbeitsmarkt hofft (Keßler und Krüger 2018). Dabei operieren die Schulen selbst in einem globalen Bildungsmarkt, indem sie nicht nur eine national gebundene Bildungsklientel anziehen oder bereits transnational organisiert sind, sondern vergleichbare andere exklusive Bildungseinrichtungen auf nationaler wie globaler Ebene beobachten und mit ihnen konkurrieren (Brooks und Waters 2015; Kenway et al. 2017).

Wenn im Folgenden in Bezug auf die Rekonstruktionen der Begriff „Internationalisierung" verwendet wird, bezieht sich dieser auf den von den Schulen selbst eingebrachten Begriff, anhand dessen herausgearbeitet werden kann, wie sie Phänomene der Inter-, Trans- und Globalisierung wahrnehmen und bearbeiten und wie sie „Internationalität“" sowie ,being international“ entwerfen und verstehen.

\section{Rekonstruktionen zur Internationalisierung exklusiver Internatsschulen}

\subsection{Sample und Methode der Untersuchung}

Die folgenden Ergebnisse stammen aus der von der Deutschen Forschungsgemeinschaft geförderten Studie „Biografische Verläufe und Berufskarrieren von Absolventinnen und Absolventen exklusiver Internatsgymnasien in Deutschland“. Die Inter- 
natsschulen zeichnen sich insbesondere dadurch aus, dass sie auf der einen Seite im reformpädagogischen, hochpreisigen privaten Segment zu verorten sind und auf der anderen Seite zu den staatlichen Internatsschulen zur Förderung von Hochbegabten gehören, die ausführliche Eignungs- und Aufnahmeverfahren durchführen, aber nur ein geringes Entgelt für Unterkunft und Verpflegung beanspruchen.

Empirische Grundlage der Ausführungen bilden Experteninterviews mit dem Leitungspersonal von den fünf Internatsschulen des Samples, in denen nicht explizit nach Internationalisierung oder Internationalität gefragt wurde. Die offenen Fragen zielten vielmehr auf Schulentwicklungsprozesse, die Schülerschaft, deren Rekrutierung und Auswahl, Kooperationspartner sowie die pädagogischen Programme ab. Die Interviews wurden im Rahmen der Felderöffnung erhoben, die mit einem jeweiligen Aufenthalt an den Schulen, teilnehmenden Beobachtungen, Führungen durch Schüler*innen sowie Interviews mit mindestens einem Mitglied der Schulleitung und weiteren Funktionsträger*innen der Schulen wie bspw. Koordinator*innen von Austauschprogrammen und Curricula verbunden war. Zudem wurde ein Gespräch mit Vertretern der Ehemaligenorganisationen geführt sowie Selbstzeugnisse der Schulen und der Schülerschaft in Form von Jahresbüchern, Schüler- oder Abiturzeitungen oder auch organisatorischen Veröffentlichungen wie der Liste der Kosten für die Unterbringung etc. erhoben. Diese Erhebungen dienten zum einen der Öffnung und Erschließung des Feldes sowie der Kontaktherstellung zu den Ehemaligen der Schule, die im Fokus der Studie stehen. Im Zuge der Auswertungen bieten die schulkulturellen Auswertungen zum anderen eine empirische Basis für Spuren der Einrichtungen, die sich in den individuellen Biografien dokumentieren (vgl. Helsper et al. 2018).

Die Rekonstruktionen der aufgenommenen und transkribierten Interviews mit den Schulleitungen und Ehemaligenvertretungen erfolgen mithilfe der Dokumentarischen Methode, die für die Auswertung von Interviews von Nohl (2017) adaptiert wurde, sodass insbesondere auch das implizite, handlungsleitende Wissen der Schulleitungen in Form von Orientierungen im Vordergrund steht. Die Gespräche, die mit dem weiteren Personal geführt, aber nicht aufgenommen, sondern in ethnografischen Protokollen festgehalten wurden, dienen als Kontextinformationen, um die organisatorischen und schulkulturellen Besonderheiten zu erfassen. Bei den Orientierungen wird in der Methodologie der Dokumentarischen Methode davon ausgegangen, dass sie das konjunktive Handlungs- und Erfahrungswissen repräsentieren, dass für die Befragten aus der Handlungspraxis emergiert, jedoch nicht unmittelbar reflexiv verfügbar, also implizit ist (vgl. Bohnsack 2007, S. 59 ff.). Insofern kann mit der Dokumentarischen Methode herausgearbeitet werden, ob die Äußerungen der Schulleitungen auf einer kommunikativen, eben auch legitimatorischen Ebene oder auf der Ebene der gemeinsamen Handlungspraktiken zu verorten sind - und wie sich diese zueinander verhalten.

\subsection{Modi der Internationalisierung}

Im Folgenden werden nun die Ergebnisse der komparativen Analyse, nämlich drei Modi der Internationalisierung von Internatsschulen, vorgestellt. Bezüglich der organisatorischen Rahmenbedingungen der Internatsschulen liegt es nahe, dass sie 
in sich ähnlich räumlich, zeitlich, sachlich und sozial aufgebaut sind und funktionieren (vgl. z.B. Kalthoff 1997). Die in dieser Studie erfassten Internatsschulen unterscheiden sich aber deutlich auf der Ebene der Trägerschaft und den damit verbundenen Strukturproblematiken. So müssen die privaten reformpädagogischen Schulen wie ein Wirtschaftsunternehmen geleitet werden, in denen die Schulgesamtleitung auch entsprechend wirtschaftlich denken und agieren muss, und zwar nicht nur in Bezug auf die Schule und ihre Gebäude, sondern auch z. B. in Bezug auf die Bewirtschaftung ganzer Grundstücke und ihrer Bebauungen oder die Sicherstellung der Finanzierung durch eine ausreichende Auslastung der Internatsplätze. Zwar müssen auch staatliche Internatsschulen ökonomischen Vorgaben halten, ihre Finanzierungsgrundlage ist jedoch durch die öffentliche Hand gedeckt, sodass sie sich hauptsächlich durch ihre schulische Arbeit und mit der Rekrutierung ausreichend geeigneter Schüler*innen legitimieren müssen. Neben zahlreichen (internats-) schulischen Strukturproblematiken ist dieser grundlegende Unterschied zentral für die Art und Weise, wie die Schulleiter Internationalität und Internationalisierung verstehen, entwerfen und bearbeiten, auch wenn die Modi zum Teil an beiden Schultypen zu finden sind, wie im Folgenden gezeigt wird.

\subsubsection{Internationalisierung als Teil einer Legitimationspraxis}

Im Falle der Internatsschule 1 wird deutlich, dass der Schulleiter das Thema nichtdeutscher Schüler*innen selbstläufig mit der Begrüßung der Interviewerin einführt und damit beginnt, bevor noch das Aufnahmegerät angestellt werden kann.

[viele Schüler kommen aus dem] Ausland? was eben heißt die Eltern wohnen in China oder Russland oder Ukraine oder in Kanada oder in Mexiko, oder in (.) Spanien und (.) ähm (.) allen diesen (.) Schülern ist gemein, dass sie (.) Eltern haben die zumindest über so viel Einkommen verfügen dass sie dieses Schulgeld hier bezahlen können, [rascheln] (.) was heißt es gibt natürlich (.) ähm einerseits eine berechtigte Anspruchshaltung, wenn wir das gleiche machen wie die öffentliche Schule dann haben wir keine Legitimation ehrlicherweise denn dann kan = ma an die öffentliche Schule gehen; also wir müssen ja Dinge anders machen als die öffentliche Schule, (.) das heißt auch besser machen als die öffentliche Schule,

(Leiter Private Internatsschule 1)

Der Schulleiter der privaten Internatsschule 1 präsentiert seine Schule und das schulische Programm folglich en passant als inter- und transnational mit einer multinationalen Schülerschaft, einem parallel angebotenen englischsprachigem Curriculum, einem Schüleraustauschprogramm im Rahmen einer transnationalen Internatsschulaustauschorganisation und weiteren von der Schule organisierten Schulaustauschen. Der Blick auf die Webseite der Schule und das Gespräch mit einem Mitarbeiter der Schule bestätigt, dass Internationalität und Transnationalität schon länger an der Schule etabliert sind und zum Portfolio der Schule gehören.

Der Umgang mit besorgten Eltern, die selbst im Ausland leben, gehört für den Schulleiter zur alltäglichen Handlungsroutine. Die multinationale Schülerschaft und das Angebot des transnationalen Curriculums, des IB, tauchen selbstläufig am Rande 
auf, während die eigentliche Problemfolie aktueller Herausforderungen der Schulentwicklung im Interviewverlauf entfaltet wird. Diese Orientierungsproblem, an dem sich der Schulleiter abarbeitet, ist das einer ,teuren Privatschule“, in der Internationalität als Zustand nur ein Bestandteil der Legitimierungspraxis gegenüber staatlichen Schulen als innovativ und superior in den positiven Gegenhorizont eingerückt wird.

Zugleich sieht sich der Schulleiter mit einer bestimmten Anspruchshaltung durch die Eltern konfrontiert, die durch das Schulgeld, welches die Eltern bezahlen müssen, u. a. Eltern, die im Ausland leben, legitimiert wird, sodass das Programm der Schule in doppelter Hinsicht legitimierungsbedürftig ist. Damit wird der zweite Bestandteil in diesem Verständnis von Internationalität deutlich, nämlich dass Internationalität als fortlaufender Prozess immer wieder in der Praxis hergestellt werden muss.

Dass die eigene schulische Praxis bei den privaten Internatsschulen besonders legitimierungs-, aber auch anerkennungsbedürftig ist, dokumentiert sich zugespitzt in der folgenden Beschreibung der Rolle transnationaler Bildungsakteure und Dachverbände.

führende Schulen, die versuchen so pff im Schulbereich innovativ? ähm kreativ, schnell, oder schneller, zu sein als andere $<$ und $=$ und $=$ und $>$ interessante Sachen zu machen? da kann man auch nicht Mitglied werden, (.) die gucken, und = und wenn sie Schulen interessant finden, laden sie die ein. (Leiter private Internatsschule 5)

Es dokumentiert sich in diesem Zitat nicht nur ebenso wie bei dem Leiter der Schule 1 die Orientierung, pädagogisch und inhaltlich vorweg gehen zu müssen, sondern auch, dass eine transnationale Organisation in diesem Feld existiert, die ihre Mitglieder selbst erwählt und damit jene als führend und progressiv in dem Feld kennzeichnet. Der Schulleiter in personam wird durch die Aufnahme in den Kreis als Person und mit seiner Arbeit ausgezeichnet. Diese Auszeichnung ist Teil der Legitimation der eigenen Schule, aber auch Teil der im Feld notwendigen (externen) Valorisierung (vgl. Sackmann 2019). Diese Anerkennung von außen speist sich dabei nicht nur aus dem eigenen Feld, sondern auch aus dem Feld der Wissenschaft und der Bildungs- bzw. Förderpolitik.

\subsubsection{Internationalisierung als Schulentwicklungsaufgabe}

Während der erste Modus der Besonderung und Legitimation nur in der Gruppe der privaten Internatsgymnasien zu finden ist, lassen sich der zweite und dritte Modus quer dazu in beiden Typen herausarbeiten, wenn auch vor dem Hintergrund unterschiedlicher Strukturprobleme. Während für den Schulleiter von Internatsschule 1 Internationalität in Form einer multinationalen Schülerschaft und transnationaler Curricula ebenso wie für die fünfte Internatsschule bereits seit langem zur schulischen Praxis gehört, stellen sich die Einführung internationaler Curricula und die Aufnahme nicht-deutscher Schüler*innen für die private Internatsschule 2 und die staatliche Internatsschule 4 als Herausforderung, aber auch als Lösung für wahrgenommene Probleme der Schulentwicklung dar. 
der an- andere Zuwachs an Komplexität; ist die Internationalität? das war vor 20 Jahren auch nich gegeben = jetzt haben wir die Internationalität $=$ und $=$ Internationalität ist erstmal ein vollkommen wertfreier Begriff. der beinhaltet extre::m viele Chancen die wir gerade in diesem Jahr = das war ein Schwerpunkt unserer Schulentwicklung (.) dieses Jahr Internationalität einfach =ähm mit einer Flucht nach vorne, = also ausnahmslos =oder vor allem als = als positiv als Zugewinn zu begreifen; (Leiter private Internatsschule 2)

„Internationalität“ stellt für den Schulleiter eine aktuelle Aufgabe und Herausforderung dar, der sich die Schule nicht freiwillig stellt. In der Fokussierungsmetapher einer ,Flucht nach vorne“ dokumentiert sich eine Wahrnehmung, Getriebener der Umstände und des Wandels von Internatsschulen zu sein, durch die die Schule zur Weiterentwicklung gezwungen ist. Zugleich wird eine Ambivalenz der Orientierungen deutlich, da diese Prozesse der Internationalisierung bereits angebahnt und beschlossen wurden und nun in den positiven Gegenhorizont eines ,Zugewinns“ gerückt werden. Vor dem Hintergrund einer vormals schwindenden Schüler*innenschaft und insbesondere auch einer finanziellen sowie personellen Krise stellt die Einführung von Internationalisierung und Transnationalisierung den Bearbeitungsmodus der Schule dar, sich auf dem nun auch global wahrgenommenen Internatsschulmarkt neu zu positionieren und ihre Existenz zu sichern. Da sich die Schule gerade im Prozess befindet, lassen sich eine Reihe von Bezügen und Verortungen auf lokaler, nationaler und globaler Ebene feststellen. So antwortet der Schulleiter zur Frage der Außenwahrnehmung:

also [Internatsschule 2] ist im Gr-= ist eher eine bodenständige; Schule, wir haben nicht so ganz diesn- (.) ich sag mal; elitären Habitus, den vielleicht- den vielleicht [Internatsschule 5], besitzt, das ist-das sieht man allein schon an der Kleidung, der Schüler? nichtdestotrotz bewegen wir uns ja im gleichen Preissegment wie das andere Schuln auch tun, (.) international siehts anders aus, also für ne britische-für ne br-= für eine Schweizer Schule (.) ja bezahl ich das drei bis-drei bis vierfache, (.) ob dann da n pädagogisches Konzept dahinter steht, das (.) wage ich jetzt mal zumindest teilweise; ähm; zu bezweifeln? trotzdem bewegen wir uns hier in nem Hochpreissegment? es ist eine gewisse Blase, hat aber auch mit der Region zu tun; (Leiter private Internatsschule 2)

Der ,elitäre Habitus“ anderer Internatsschulen dient hier als Distinktionsfolie, also als negativer Gegenhorizont, der als Vergleichsrahmen in die Argumentation für die Bodenständigkeit seiner Schule eingebettet ist. Der Schulleiter versucht diese Bodenständigkeit weiter auszuführen und stellt einen Zusammenhang mit der Kleidung seiner Schüler*innen her. Der Zweifel, den er im Gespräch äußert, dass hinter noch deutlich teureren Internatsschulen wie in der Schweiz oder Großbritannien ein pädagogisches Konzept stünde, kann als Positionierung gelesen werden. Diese Lesart bestätigt sich, wenn er im Fortgang betont, dass die Wohnstandards in britischen Internaten sehr niedrig seien, aber es dort in Kauf genommen würde, da es sich um ein ,,anderes Produkt“ handele: ,wenn Sie sich da die Unterkünfte angucken; (.) die sind bei weitem- haben die nicht den Standard den $=$ Sie $=$ den $=$ Sie jetzt hier haben, 
(.) aber dort nimmt man es vielleicht eher in Kauf, weil das P:rodukt eigentlich auch ein ganz anderes ist." (Leiter private Internatsschule 2).

Das „,andere Produkt“ ist aus Sicht des Schulleiters eben kein pädagogisches Konzept, sondern das Renommee und die Möglichkeit des Zugangs zu Eliteuniversitäten, die der Abschluss an britischen Internaten erbringt. Dabei lässt er außer Acht, dass die Form der spartanischen, kasernenartigen - nicht familienähnlichen - Unterbringung durchaus als eine bestimmte Erziehung, z. B. im Sinne einer Disziplinierung verstanden werden kann. Die lokale Verortung wird mit der betonten Nähe zu einer deutschen Metropole vorgenommen. Die gesteigerte Anforderung an die Wohnstandards des Internats erklärt er mit der Nähe zu dieser Metropole, aus der auch traditionell ein Großteil der Klientel stammt und an deren Standards sich die Internatsschule anpassen müsse, um attraktiv zu bleiben. Insofern ist Schulentwicklung nicht nur im Sinne inhaltlich-pädagogischer Programme zu verstehen, sondern auch in Bezug auf die „Markenentwicklung“.

Für die beiden staatlichen Internatsschulen liegen aufgrund der staatlichen Finanzierungen derartige Positionierungen nicht im Problemhorizont. Dennoch sind auch bei diesen Schulleitern Handlungspraktiken zu rekonstruieren, die auf eine wahrgenommene Anforderung der inhaltlich-curricularen Profilierung im Kontext der Schulentwicklung bezogen sind. Obwohl die staatliche Internatsschule 3 keine international anerkannten Abschlüsse vergibt, wird das Thema im Bereich des bilingualen Unterrichts virulent.

sind wir nicht den großen Schritt gegang und habm gesagt wir (.) bietn den Unterricht in bestimmtn Fächern über das ganze Jahr in der Fremdsprache an (.) sondern wir ham vielmehr versucht dort wo es sich für die Unterrichtseinheit anbietet (.) dann Englisch als Unterrichtssprache einzusetzn. (.) nenn mal ein Beispiel, im Geografieunterricht wenn man Australien behandelt, bietet es sich natürlich an (.) das in Englisch zu tun. in Geschichte wenn die Geschichte Amerikas besprochn wird ebenfalls. (.) es wäre aber glau:be:, der falsche Schritt (.) wenn man (.) die deutsche Geschichte beispielsweise des zwanzigstn Jahrhunderts jetzt auf Englisch unterrichtn würde. das macht wenig Sinn. wir sind da durchaus auch experimentell, (Leiter staatliche Internatsschule 3)

Die Darstellung dokumentiert einen schulinternen Versuch der Ausweitung bilingualer Unterrichtseinheiten, der in der Summe dazu geführt hat, dass der Unterricht nicht generell umgestellt wurde. Der Allsatz ,wir sind da durchaus auch experimentell" ist auf der Ebene der Legitimation zu verorten, da die Exemplifikationen darauf hinweisen, dass eine flächendeckende oder zumindest systematische Bilingualität nicht eingeführt wurde. Die eher resignierenden Formulierungen ,ham vielmehr versucht" sowie ,wir hams [...] versucht" dokumentieren, dass die Idee einer solchen Einführung bereits abgeschlossen und keine Fortführung oder Ausweitung möglich ist. Es liegt also im persönlichen Ermessen der Lehrkräfte, ob sie eine bestimmte Unterrichtseinheit in einer anderen Sprache anbieten. Damit gehört Bilingualität nicht zur Alltagspraxis, existiert aber weiter als positiver Gegenhorizont (,eine sehr willkommene Abwechslung“). Im Orientierungsrahmen des Schulleiters wird auch deutlich, dass fremdsprachlicher Fächerunterricht auf Unterrichtseinhei- 
ten, die etwas mit dem „Stammland“ der Sprache zu tun haben, begrenzt bleiben müsse.

Die staatliche Internatsschule 4 bietet dagegen ein transnationales Curriculum und den entsprechenden Abschluss an, unter anderem auch, da das fremdsprachliche Curriculum zum schulischen Profil gehört und nachgefragt wird:

Internationalisierung; (.) das war ein Ursprungskonzept überhaupt (.) und die Frage war immer ein bisschn m- wie macht man das wie kriegt man hier auch dann: so ne Mehrsprachigkeit eben rein, (.) dann: (2) ${ }^{\circ}$ ich weiß jetz auch nich wie der internationale Abschluss dann wieder aufgetaucht is: ${ }^{\circ}$ also letztnendes war = s für uns dann $n$ bisschn die Frage wo:: liegn eigenlich jetz noch Herausforderungn welche Herausforderung: kann: ich noch entwickeln um: diesn Schülern auch Futter: zu geben dass sie (.) sich daran richtig reiben können und wirklich gut zu tun das is nich jedermanns Sache äh in=nem irgenwie 35 Prüfungn zu machn (.) weil=er [zwei Abschlüsse] macht (Leiter staatliche Internatsschule 4)

Es dokumentiert sich ähnlich wie bei dem Leiter der Internatsschule 3 ein enges Verständnis von mehrsprachigem Unterricht als Internationalität. Das Verständnis dieses Schulleiters meint ausschließlich eine curriculare Besonderheit, die eigentlich eine Zweisprachigkeit ist, und wie die Schüler*innen, die sich durch eine besondere Leistungsstärke auszeichnen, ,befriedigt“" werden können. Dadurch entsteht auch eine zusätzliche Leistungsdifferenzierung zwischen jenen, die das Abitur und den international anerkannten Abschluss absolvieren, und einer Restkategorie. Die Hereinnahme des zusätzlichen Abschluss stellt eine Maßnahme der eigenen Schulentwicklungspraxis dar, die sich vor dem positiven Gegenhorizont einer stetigen Weiterentwicklung artikuliert. Diese stetige Weiterentwicklung gilt es nicht nur in Form eines Angebots für die Schülerschaft auszubauen und in Form des Anspruchs an die Schülerschaft, sich weiterzuentwickeln, sondern adressiert auch das Lehrpersonal. Denn durch das Programm stehen dem Schulleiter nun Mittel zur Verfügung, die Lehrerschaft zur Fortbildung zu verpflichten und strukturelle Anreize schaffen zu können. Dabei erhebt er hohe Ansprüche (unabhängig von einer Hochbegabung), außergewöhnlich und inspirierend zu unterrichten, da es sich für ihn um eine besondere Schule handelt. Damit wird die Anspruchshaltung, die in den Ausleseverfahren an die Schüler*innen angelegt wird, auch an die Lehrkräfte herangetragen.

\subsubsection{Internationalisierung als Rekrutierungsressource}

Der dritte Modus, wie die Schulleiter Internationalisierung innerhalb ihrer Orientierungsrahmen verstehen, entwerfen und bearbeiten, lässt sich ebenfalls an privaten wie staatlichen Internatsschulen finden. So geht aus einem Gespräch mit dem stellvertretenden Schulleiter der privaten Internatsschule 2 hervor, dass die Aufnahme von nicht-deutschen Schüler*innen den Vorteil hat, dass es sich dabei um eine zahlungskräftige Klientel handelt, die mindestens ein Jahr im Voraus die Jahressumme überweist. Allerdings dürfe der Anteil der chinesischen und russischen Schüler*innen nicht überhand nehmen, da sonst Zustände wie an ,Schweizer Internaten“ drohen würden, wenn Schüler*innen mehrheitlich aus Ländern kämen, die die 
freiheitlich-demokratischen Grundwerte nicht teilen würden. Der Schulleiter thematisiert dagegen im Interview die systematische Steuerung der Bewerberauswahl, die er in Kooperation mit einer ,also ich tu mich mit dem Begriff Elite unheimlich schwer; ja? aber in dem Falle fällt mir einfach nichts Besseres ein--, einer Elitesprachschule, in China?" trifft, in dem diese geeignete Bewerber*innen einlädt. In den Gesprächen versucht er, ,die Topabsolventen nach Gesprächen zu bekommen, (.) und in den Gesprächen (.) versuchen wir (.) zu ermitteln = gemeinsam mit den Eltern ob der Schüler auch wirklich passt. was ist passend, bei einem chinesischen Schüler? es muss diese gewisse Offenheit da sein;““. Dabei scheint neben dem Kriterium der Bestenauswahl anhand von Noten und Sprachkenntnissen das vage Kriterium der „Offenheit“" ein entscheidendes zu sein.

Neben der sich dokumentierenden aufwändigen Auswahl vor Ort, die die multinationale Zusammensetzung der Schülerschaft in einer für die Schule und die Schüler*innenschaft förderlichen Weise absichern soll, ist die Auswahl am Beispiel der chinesischen Schüler*innen aus mehreren Gründen wichtig. Dabei wird die Übereinstimmung mit den freiheitlich-demokratischen Grundwerten nur indirekt mit den Codes der „Passung“ und der „Offenheit" thematisiert, während die akademische Leistungsstärke offen betont wird, indem die nicht-deutschen Schüler*innen als „Zugpferde“ für deutsche Schüler*innen dienen sollen, aber auch als Sicherheit, dass sie die Ansprüche des deutschen Abiturs mit den entsprechenden sprachlichen Fähigkeiten auch erfüllen können (und damit auch die erfolgreiche Arbeit der Schule bestätigen). Der Aspekt der Schülerkomposition rückt also deutlich in den Fokus sowie der Versuch, über die aktive Gestaltung der Auswahl diese zu steuern. Nach dem Anteil der nicht-deutschen Schüler*innen gefragt, geht der Schulleiter entsprechend auch mit seinen Worten auf die „Durchmischung“ ein, die im Internat bei $40 \%$ und in der Schule durch den Anteil der Tagesschüler bei $20 \%$ läge, was wichtig sei, da es sonst schwierig sei, „Gymnasialniveauunterricht“ durchzuführen.

Der Schulleiter der staatlichen Internatsschule 4, der ebenso wie die andere staatliche Schule nur Schüler*innen aus Deutschland rekrutieren kann, weist dennoch eine Orientierung an einer multinationalen Zusammensetzung der Schülerschaft auf, die durch die organisationalen Rahmungen begrenzt sind. So betont er, dass dies zwar begrenzt über Schüler*innenaustausche mit Partnerschulen, aber aufgrund der Bindung des Internats an die Unterrichtszeiten generell nicht möglich ist. Durch die Einführung eines transnationalen Curriculums und Abschlusses häuften sich nun an dieser Schule jedoch Anfragen von „Chinesen“ und „Russen“, die mit dem Verweis auf die nicht vorhandene außerunterrichtliche Unterbringung abgewiesen werden müssten. Aber statt auf die Tatsache eines staatlichen steuerfinanzierten Internats hinzuweisen, werden das Betreuungsproblem und die fehlende sprachlichen Kompetenzen der Bewerber*innen in Anschlag gebracht. Dies lässt auf einen Orientierungsrahmen schließen, in dem grundsätzlich „Hochbegabte“ gleich welcher Nation aufgenommen werden können, der allerdings mit Essenzialisierungen einhergeht:

ich hatte jetzt ma: (.) da meldet sich mal: $n$ Russe: und=so: und der is aber ${ }^{\circ}()<$. dann auch schnell wieder durch $=s$ Auswahlverfahren gefallen, weil wir natürlich im Auswahlverfahren das is die Krux an=der ganzen Angelegenheit ja au:ch> (.) immer ein auf die deutsche Sprache bezogenes Auswahl- 
verfahren machen; ${ }^{\circ}$ und das is ${ }^{\circ}$ (2) also das is dann: (.) scho:n auch sehr schwe:r für jemandn der aus = $m$ Ausland kommt; da durchzukommen (.) (Leiter staatliche Internatsschule 4)

Die gegebenen formalen Rahmenbedingungen und die konzeptionell beanspruchte Internationalität der Schule sind auf in den Handlungsorientierungen gekoppelt mit dem Angebot eines mehrsprachigen Unterrichts, das exklusiv deutschen Schüler*innen vorbehalten ist, aber das transnationale Bildungsprogramm des international anerkannten Abschlusses nicht als solches gedeutet wird.

\subsection{Zusammenfassung}

Insgesamt lässt sich feststellen, dass die Praxis von Internationalisierung sowie der Umgang mit transnationalen Organisationen und Angeboten zwar auch der Legitimation und Besonderung von insbesondere privaten Internatsschulen dienen, zugleich konnten jedoch noch zwei weitere Modi der Internationalisierung herausgearbeitet werden, die zum einen die innerschulische Weiterentwicklung und zum anderen die Rekrutierungs- und Kompositionspraktiken der Schulen widerspiegeln.

Im Unterschied zu den privaten Internatsschulen 1 und 5 stellt Transnationalität und Internationalisierung für den Leiter der Internatsschule 2 eine aktuelle Aufgabe und Herausforderung dar, sodass der Internationalisierungsprozess der Schule in statu nascendi erfasst werden konnte. Bei den privaten Schulen ist zudem eine Entwicklung zur ,Versäulung“ mit der Einführung eigener Grundschulen, international anerkannten Schulabschlüssen bzw. transnationalen Vernetzungen und mehreren angebotenen Bildungsgängen gemeinsam (vgl. Helsper et al. 2016, S. 710). Dass die international anerkannten Curricula und Abschlüsse in deutschen Internatsschulen auch stark durch russische und chinesische Eltern nachgefragt werden, weist auf ihre Attraktivität im Sinne eines distinktiven Markierers und einer positiven Privilegierung auch außerhalb von Deutschland hin. Die Wahrnehmung einer Anforderung, zum einen transnationale Bildungsprogramme anzubieten und zum anderen eine multinationale Schülerschaft zu ,komponieren“, bzw. mit diesen einen angemessenen pädagogischen Umgang zu finden, zeigt sich in den privaten Internatsschulen in verschärfter Art und Weise. Nicht-deutsche Schüler*innen stellen eine wichtige Einnahmequelle dar und sind repräsentativ für das Selbst-Marketing, solange kulturelle und nationale Diversität als Bereicherung und Profilmerkmal wahrgenommen werden können.

Hingegen sind den staatlichen Schulen durch die staatliche Finanzierung, die curricularen Vorgaben und die Beschränkung auf eine vorrangig nationale Schüler*innenschaft in Bezug auf transnationale Kooperationen und Beziehungen enge Grenzen gesetzt. Nichtsdestotrotz konkurrieren auch staatliche Internatsschulen um eine geeignete Schüler*innenschaft, weshalb Internatsschule 4 den internationalen Abschluss auch aus Gründen der Attraktivität eingeführt hat. Für private Internatsschulen scheint sich diesbezüglich aber ein deutlich höherer Profilierungsbedarf abzuzeichnen, zumal hier auch ausländische Internatsschulen als Referenzrahmen und potenzielle Mitbewerber im Horizont stehen. 


\section{Fazit}

In den Rekonstruktionen zeigt sich, dass Internationalität als stratifikatorische und legitimatorische Ressource im Feld der Internatsschulen an Bedeutung gewinnt. Internationalität wird insbesondere von den Leitern der Schulen 2 und 4 als anzustrebende Ressource und Praxis wahrgenommen, sich gegenüber Mitbewerbenden aus dem nationalen, im Fall der privaten Internatsschule aber auch den im Ausland liegenden Internatsschulen, und exklusiven Tagesgymnasien in der Region zu behaupten. Insofern scheinen sich insbesondere private Internatsgymnasien einem internationalen (insbesondere anglophonen) Schulmarkt anzugleichen, um wettbewerbsfähig zu bleiben. Darüber hinaus werden die Schulen selbst zu Agenten der Internationalisierung, die sie mit vorantreiben. In den Prozess ihrer Legitimierung als besondere Schulen (im Sinne von kreativ und innovativ und damit aber auch als superior und exzellent) (vgl. Maxwell 2018, S. 348) sind im privaten Bereich transnationale Dachverbände und Organisationen involviert, während für die staatlichen Internatsschulen hauptsächlich staatliche Ministerien an der Valorisierung, aber auch die im Sample befindlichen privaten Schulen durch die Etablierung von Netzwerken privater und staatlicher Internatsschulen beteiligt sind.

Bezüglich der Befunde zu Internatsschulen im anglophonen Raum zeigen sich Parallelen, die sich in einer Gleichzeitigkeit der Betonung von Internationalität einerseits und der Verbundenheit mit der Region und den lokalen Kommunen bzw. Gemeinden andererseits manifestieren (vgl. Maxwell 2018, S. 362; Kenway et al. 2017). Auch die drei privaten Internatsschulen pflegen eine komplexe Verbindung, gleichzeitig internationale Bezüge und transnationale Beziehungen herzustellen und auf die wichtige Zusammenarbeit mit lokalen Dienstleistern, den Eltern und der Bevölkerung hinzuweisen. Projekte, wie die Organisation von Flüchtlingshilfen vor Ort oder die sozialen Dienste, die die Schüler*innen leisten, stellen eine Verbindung zur lokalen Umgebung her und damit auch ein Einverständnis der Bevölkerung mit der Einrichtung vor Ort (in Teilen auch - jedoch ohne internationalen Bezug - die staatlichen Internatsschulen) (vgl. Courtois 2016; Kenway et al. 2017; Howard 2018). So zeigt sich, dass die distinktive und die legitimatorische Funktion in den Praktiken eng verwoben sind, indem die Schüler*innen soziale sowie internationale Kontakte und Kompetenzen mit ihresgleichen erwerben, womit zugleich die Attraktivität der Internatsschulen steigt, die diesen Rahmen bieten.

In dem Zusammenhang zwischen dem gleichzeitigen Engagement mit dem Globalen und dem Lokalen arbeitet Maxwell (2018, S. 362) mehrere Widersprüche heraus, die mit den verschiedenen Praktiken der Internationalisierung einhergehen und die auch auf die Internatsschulen zutreffen. Neben dem Befund, dass es offensichtlich negativ wie positiv privilegierende Formen von Internationalisierung gibt, stellt Maxwell fest, dass es sich bei den meisten Umsetzungen von Internationalität um Praktiken der Internationalisierung ,at home“ handelt, was letztlich auf eine legitimatorische Funktion hinweist (Maxwell 2018, S. 365). Dies trifft auf viele der exklusiven Tagesgymnasien (Helsper et al. 2016; Keßler und Krüger 2018; Kotzyba et al. 2018) mit Ausnahme der International Schools zu, bei den hier vorgestellten Internatsschulen jedoch lediglich auf die staatlichen und bei diesen insbesondere auch aufgrund ihrer rechtlichen Beschränkungen. Da sich die staatlichen Hochbegab- 
teninternatsschulen mit den untersuchten privaten Internatsschulen austauschen und diese als Innovatoren anerkennen, steht zu vermuten, dass sich ohne diese rechtlichen Beschränkungen Tendenzen zur Strukturangleichung im Sinne der herausgearbeiteten Internationalität als legitimatorischer Ressource und als herzustellendes Produkt auch bei den staatlichen Internatsschulen durchsetzen würden. Internationalisierung und transnationale Bedingungen wie Beziehungen sind insbesondere für die privaten Schulen im Sinne einer Legitimierung und Besonderung überlebensnotwendig, im Kontext von Schul- und Curricula-Entwicklung sowie der Schülerrekrutierung auch für die staatlichen Schulen.

Die positiven Privilegierungen, die in diesem Segment zu finden sind, sind neben dem Superioritätsausweis der Schulen die Komposition einer - im privaten Bereich - internationalen und kosmopolitischen Peergemeinschaft, in der durch den Aufenthalt per se schon interkultureller Austausch stattfindet (so die Orientierungen) und - im staatlichen Bereich - einer leistungsbezogen ausgewählten, homogenisierten Schülerschaft, die durch die Sprachenprofilierung, aber auch die Zugzwänge der Normalitätsvorstellung von Auslandsaufenthalten ebenfalls zu einer transnationalen Mobilität der Bildungsbiografien - wie die Studie im Hinblick auf die Ehemaligen zeigt - beitragen. Die Schüler*innen der privaten Internatsschulen werden jedoch nicht nur durch die multinationalen, jedoch sozio-ökonomisch weitgehend homogene Zusammensetzung der Schülerschaft positiv privilegiert oder durch den Ruf der Schulen, sondern auch durch die vielfältigen Angebote an Austauschmöglichkeiten mit den im Netzwerk befindlichen Internatsschulen im Ausland, durch die diesbezüglichen individuellen Beratungsangebote sowie der sprachlichen und curricularen Zusatzangebote. Damit wird mit der Teilnahme an den Curricula und Austauschprogrammen nicht nur symbolisches und kulturelles Kapital erworben, sondern auch soziales Kapital in Form von Kontakten und Freundschaften aufgebaut (vgl. Gerhards et al. 2016; Keßler et al. 2018). In diesem Kontext ist dann auch eine „Erwählung“ durch staatliche Hochbegabtenschulen positiv privilegierend, da auch diese Form der Bildung als distinktive Markierer des Konsums und eines besonderen Lebensstils wahrgenommen werden, wie auch das Interesse nicht-deutscher Eltern zeigt. Der Zugang zu englischsprachigen Universitäten wird insbesondere bei den privaten Internatsschulen durch Beratungsangebote gewährleistet (vgl. Krüger et al. 2019 für International Schools), deren Anbieter*innen die Spielregeln des europäischen und anglophonen Hochschulsegments kennen und dieses Wissen zur Verfügung stellen.

Förderung Das Forschungsprojekt „Biografische Verläufe und Berufskarrieren von Absolventinnen und Absolventen exklusiver Internatsgymnasien in Deutschland" wird von der Deutschen Forschungsgemeinschaft seit 2017 mit einer Sachbeihilfe gefördert (Kennzeichen: DE 2811/1-1).

Funding Open Access funding provided by Projekt DEAL.

Open Access Dieser Artikel wird unter der Creative Commons Namensnennung 4.0 International Lizenz veröffentlicht, welche die Nutzung, Vervielfältigung, Bearbeitung, Verbreitung und Wiedergabe in jeglichem Medium und Format erlaubt, sofern Sie den/die ursprünglichen Autor(en) und die Quelle ordnungsgemäß nennen, einen Link zur Creative Commons Lizenz beifügen und angeben, ob Änderungen vorgenommen wurden.

Die in diesem Artikel enthaltenen Bilder und sonstiges Drittmaterial unterliegen ebenfalls der genannten Creative Commons Lizenz, sofern sich aus der Abbildungslegende nichts anderes ergibt. Sofern das betref- 
fende Material nicht unter der genannten Creative Commons Lizenz steht und die betreffende Handlung nicht nach gesetzlichen Vorschriften erlaubt ist, ist für die oben aufgeführten Weiterverwendungen des Materials die Einwilligung des jeweiligen Rechteinhabers einzuholen.

Weitere Details zur Lizenz entnehmen Sie bitte der Lizenzinformation auf http://creativecommons.org/ licenses/by/4.0/deed.de.

\section{Literatur}

Adick, C. (2008). Transnationale Bildungsorganisationen in transnationalen Bildungsräumen: Begriffsdefinitionen und Vorschlag für eine Typologie. Tertium Comparationis, 14(2), 168-197.

Adick, C. (2018). Transnational education in schools, universities, and beyond: Definitions and research areas. Transnational Social Review, 8(2), 124-138.

Angod, L., \& Gaztambide-Fernández, R. (2019). Endless land, endless opportunity. The coloniality of elite boarding school landscapes. Zeitschrift für Pädagogik, 65(2), 227-241.

Ball, S. J. (2012). Global education inc. Oxon: Routledge.

Ball, S. J., \& Nikita, D. P. (2014). The global middle class and school choice: A cosmopolitan sociology. In H.-H. Krüger \& W. Helsper (Hrsg.), Elite und Exzellenz im Bildungssystem: Nationale und internationale Perspektiven (Zeitschrift für Erziehungswissenschaft: Sonderheft 19, S. 81-93). Wiesbaden: Springer VS.

Bauman, Z. (1998a). On glocalization: Or globalization for some, localization for some others. Thesis Eleven, $54,37-49$.

Bauman, Z. (1998b). Work consumerism and the new poor. Buckingham: Open University Press.

Bellmann, J. (2008). Choice Policies - Selektion, Segregation und Distinktion im Rahmen von Bildungsmärkten. In H. Ullrich \& S. Strunck (Hrsg.), Begabtenförderung an Gymnasien (S. 249-270). Wiesbaden: VS.

Böhme, J. (2000). Schulmythen und ihre imaginäre Verbürgung durch oppositionelle Schüler. Bad Heilbrunn: Klinkhardt.

Bohnsack, R. (2007). Rekonstruktive Sozialforschung (6. Aufl.). Opladen: Budrich.

Boli, J. (2006). World polity theory. In R. Robertson \& J. A. Scholte (Hrsg.), Encyclopedia of globalization (Bd. 1). London: Routledge.

Brooks, R., \& Waters, J. (2015). The boundaries of privilege: Elite English schools' geographies and depictions of a local community. In A. van Zanten, S. J. Ball \& B. Darchy-Koechlin (Hrsg.), World yearbook of education 2015 (S. 95-107). London: Routledge.

Courtois, A. (2016). Internationalising practices and representations of the "other" in second-level elite schools in Ireland. Globalisation, Societies and Education, 14(4), 560-576.

Deppe, U. (2019). Nur eine Episode? Überlegungen zur Untersuchung von ehemaligen Internatsgymnasiasten und der Bedeutung des Internatsbesuchs. Zeitschrift für Pädagogik, 65(2), 212-226.

Deppe, U., \& Kastner, J. (2014). Exklusive Bildungseinrichtungen in Deutschland. In H.-H. Krüger \& W. Helsper (Hrsg.), Elite und Exzellenz im Bildungssystem (Zeitschrift für Erziehungswissenschaft: Sonderheft 19, S. 263-283). Wiesbaden: Springer VS.

Deppe, U., Lüdemann, J., \& Kastner, H. (2018a). Processes of internationalisation, stratification and elite formation in the German education system. In C. Maxwell, U. Deppe, H.-H. Krüger \& W. Helsper (Hrsg.), Elite education and Internationalisation (S. 309-330). Houndmills: Palgrave Macmillan.

Deppe, U., Maxwell, C., Krüger, H.-H., \& Helsper, W. (2018b). Elite Education and Internationalisation From the Early Years into Higher Education. An Introduction. In C. Maxwell, U. Deppe, H.-H. Krüger \& W. Helsper (Hrsg.), Elite education and Internationalisation (S. 1-22). Houndmills: Palgrave Macmillan.

Gaztambide-Fernández, R. (2009). What Is an Elite Boarding School? Review of Educational Research, 79(3), 1090-1128.

Gerhards, J., Hans, S., \& Carlson, S. (2016). Klassenlage und transnationales Humankapital. Wiesbaden: Springer VS.

Gibson, A. (2017). Klassenziel Verantwortungselite. Wiesbaden: Springer VS.

Guarnizo, L.E., \& Smith, M.P. (1998). The Locations of Transnationalism. In L.E. Guarnizo \& M.P. Smith (Hrsg.), Transnationalism from Below (S. 3-34). New Brunswick: Transaction Publishers.

Hayden, M., \& Thompson, J. (Hrsg.). (2016). International schools: Current Issues and Future Prospects. Oxford: Symposium Books. 
Helsper, W., Kramer, R.-T., Böhme, J., \& Lingkost, A. (2001). Schulkultur und Schulmythos. Opladen: Leske + Budrich.

Helsper, W., Dreier, L., Gibson, A., Kotzyba, K., \& Niemann, M. (2015). „Exklusive“ und private Gymnasien in städtischen Bildungsregionen. Wettbewerb und Schülerauswahl am städtischen höheren Bildungsmarkt. In M. Kraul (Hrsg.), Private Schulen (S. 45-61). Wiesbaden: VS.

Helsper, W., Krüger, H.-H., Dreier, L., Keßler, C.I., Kreuz, S., \& Niemann, M. (2016). International orientierte höhere Schulen in Deutschland - Zwei Varianten von Internationalität im Wechselspiel von Institution und Schülerbiografie. Zeitschrift für Erziehungswissenschaft, 19(4), 705-725.

Helsper, W., Dreier, L., Gibson, A., Kotzyba, K., \& Niemann, M. (2018). Exklusive Gymnasien und ihre Schüler Passungsverhältnisse zwischen institutionellem und individuellem Schülerhabitus. Wiesbaden: Springer VS.

Hinrichsen, M., \& Paz Matute, P. (2018). „Den Horizont erweitern?“ - Schulische Internationalisierung und die Konstruktion (trans-)nationaler Bildungsräume. Tertium Comparationis, 24(2), 190-205.

Hornberg, S. (2010). Schule im Prozess der Internationalisierung von Bildung. Münster: Waxmann.

Hornberg, S. (2018). Transnationale Bildungsräume am Beispiel von IB World Schools. In E. Glaser, H.C. Koller, W. Thole \& S. Krumme (Hrsg.), Räume für Bildung - Räume der Bildung (S. 226-233). Leverkusen: Barbara Budrich.

Howard, A. (2018). Making It Political: Working Towards Transformation in the Study of Internationalisation of Elite Education. In C. Maxwell, U. Deppe, H.-H. Krüger \& W. Helsper (Hrsg.), Elite education and Internationalisation (S. 331-345). Houndmills: Palgrave Macmillan.

Hummrich, M. (2018). Transnationalisierung, Transnationalität und der Vergleich von Schulkulturen. Tertium Comparationis, 24(2), 171-189.

International Baccalaureate Organization (2019). Find an IB World School. https://www.ibo.org/pro grammes/find-an-ib-school/. Zugegriffen: 26. Mai 2019.

Kalthoff, H. (1997). Wohlerzogenheit. Eine Ethnographie deutscher Internatsschulen. Frankfurt a. M.: Campus.

Kenway, J., Fahey, J., Epstein, D., Koh, A., McCarthy, C., \& Rizvi, F. (2017). Class choreographies: Elite schools and globalization. Basingstoke: Palgrave Macmillan.

Kenway, J., Fahey, J., \& Koh, A. (2013). The libidinal economy of the globalising elite school market. In C. Maxwell \& P. Aggleton (Hrsg.), Privilege, agency and affect. Understanding the production and effects of action (S. 15-31). New York: Palgrave Macmillan.

Keßler, C. I., \& Krüger, H.-H. (2018). "Being International": Institutional Claims and Student Perspectives at an Exclusive International School. In C. Maxwell, U. Deppe, H.-H. Krüger \& W. Helsper (Hrsg.), Elite education and Internationalisation (S. 209-228). Houndmills: Palgrave Macmillan.

Keßler, C. I., Kotzyba, K., \& Schippling, A. (2018). Biografieanalyse und transnationales Kapital - Theoretische und methodologische Reflexionen. Tertium Comparationis, 24(2), 228-245.

Knight, J. (2004). Internationalization Remodeled: Definition, Approaches, and Rationales. Journal of Studies in International Education, 8(1), 5-31

Kotzyba, K., Dreier, L., Niemann, M., \& Helsper, W. (2018). Processes of Internationalisation in Germany's Secondary Education System. In C. Maxwell, U. Deppe, H.-H. Krüger \& W. Helsper (Hrsg.), Elite education and Internationalisation. From the early years to higher education (S. 191-208). Houndmills: Palgrave Macmillan.

Krüger, H.-H., Schanze, A., \& Winter, D. (2019). Zwischen Eliteuniversität und dualer Berufsausbildung. In H.-H. Krüger, K. Hüfner, C. Keßler, S. Kreuz, P. Leinhos \& D. Winter (Hrsg.), Exklusive Bildungskarrieren von Jugendlichen und ihre Peers am Übergang in Hochschule und Beruf (S. 259-283). Wiesbaden: Springer.

Maxwell, C. (2018). Changing Spaces-The Reshaping of (Elite) Education Through Internationalisation. In C. Maxwell, U. Deppe, H.-H. Krüger \& W. Helsper (Hrsg.), Elite education and Internationalisation (S. 347-367). Houndmills: Palgrave Macmillan.

Meyer, J.W. (2005). Weltkultur. Wie die westlichen Prinzipien die Welt durchdringen. Frankfurt a. M.: Suhrkamp.

Nohl, A.-M. (2017). Interview und Dokumentarische Methode (5. Aufl.). Wiesbaden: Springer VS.

Ong, A. (1999). Flexible citizenship: The cultural logics of transnationality. Durham, NC: Duke University Press.

Pfaff, N. (2018). Erziehungswissenschaftliche Transnationalismusforschung im Gegenstandsbereich der Schule - zwischen Struktur und Lebenswelt. Tertium Comparationis, 24(2), 151-170.

Pries, L. (2010). Transnationalisierung. Theorie und Empirie grenzüberschreitender Vergesellschaftung. Wiesbaden: Springer VS. 
Prosser, H. (2018). Elites Go Public? International Baccalaureate's Decolonising Paradox in Ecuador. In C. Maxwell, U. Deppe, H.-H. Krüger \& W. Helsper (Hrsg.), Elite education and Internationalisation (S. 229-245). Houndmills: Palgrave Macmillan.

Resnik, J. (2016). The development of the International Baccalaureate in Spanish speaking countries: a global comparative approach. Globalisation, Societies and Education, 14(2), 298-325.

Sackmann, R. (2019). Mechanismen der Elitebildung: Theoretisches Konzept und empirische Trends im deutschen Bildungssystem und im internationalen Vergleich. In W. Helsper \& H.-H. Krüger (Hrsg.), Exklusive Bildung und neue Ungleichheit (Zeitschrift für Pädagogik: Beiheft 65, S. 41-60). Weinheim: Beltz Juventa.

Spring, J. (2015). Globalization of education. New York: Routledge.

Stronach, I. (2010). Globalizing education, educating the local. London: Routledge.

Ullrich, H. (2014). Exzellenz und Elitenbildung in Gymnasien. Traditionen und Innovationen. In H.H. Krüger \& W. Helsper (Hrsg.), Elite und Exzellenz im Bildungssystem (Zeitschrift für Erziehungswissenschaft: Sonderheft 19, S. 181-202). Wiesbaden: Springer VS.

Waldow, F. (2018). Commentary to Part III. Why Is "Being International" So Attractive? In C. Maxwell, U. Deppe, H.-H. Krüger \& W. Helsper (Hrsg.), Elite education and Internationalisation (S. 247-252). Houndmills: Palgrave Macmillan.

Weber, M. (2002). Wirtschaft und Gesellschaft: Grundriss der verstehenden Soziologie. Tübingen: Mohr Siebeck.

Weiss, A. (2005). The transnationalization of social inequality. Current Sociology, 53(4), 707-728.

Yemini, M. (2015). Internationalisation discourse hits the tipping point. Perspectives: Policy and Practice in Higher Education, 19(1), 19-22.

Yildiz, E. (2018). Vom methodologischen Nationalismus zu postmigrantischen Visionen. In M. Hill \& E. Yildiz (Hrsg.), Postmigrantische Visionen (S. 43-62). Bielefeld: transcript.

Zymek, B. (2016). Stichwort: Internationalisierungsprozesse und Elitebildung. Zeitschrift für Erziehungswissenschaft, 19(4), 671-688. 